2 NHS England. Serious Incident Framework: Supporting Learning to Prevent Recurrence. NHS England, 2015.

3 Health and Social Care Act 2008 (Regulated Activities) Regulations 2014: Regulation 20 Duty of Candour. Available at: http:// www.cqc.org.uk/content/regulation-20-duty-candour\#legislation-links

doi: $10.1192 / p b .41 .5 .297$

\section{Management of common mental disorders for psychogeriatric patients in Hong Kong - a comparison of two clinics}

The enhanced Common Mental Disorder Clinic (CMDC) was developed by the Hospital Authority of Hong Kong in 2015 to shorten new-case waiting time at the psychiatric specialist out-patient clinic (SOPC) for adult patients with common mental disorders (CMDs). ${ }^{1}$ Patients with CMDs usually have to wait for a long time for their first psychiatric consultation and during that period symptoms may worsen and become more distressing. They may require a longer duration of treatment if their problems have become more complicated. CMDC aims at providing early treatment to nip the problem in the bud. It consists of two doctors, three nurses, two occupational therapists and one clinical psychologist. Each patient is under the care of a multidisciplinary team. The additional allied health input aims to reduce doctors' burden. It is hoped that after one year of treatment, a substantial number of patients can be discharged from the programme.

CMDC started its operation in the Kowloon East Cluster of Hong Kong in July 2016 - Kowloon East is one of seven clusters governed by the Hospital Authority and it serves a population size of about 1.1 million. Psychogeriatric patients are normally seen at SOPC, with waiting times of 110 weeks in 2015. We intend to include psychogeriatric patients in the new programme with a view to shorten the waiting time.

A pilot study has been conducted by recruiting 30 consecutive psychogeriatric patients, with CMDs, from CMDC and SOPC retrospectively. The difference in clinical factors between the two groups, as well as any benefit of shortening the waiting time by triaging suitable psychogeriatric patients from SOPC to CMDC, were assessed. Aims of the pilot programme were to demonstrate any benefit of extending CMDC to psychogeriatric patients and to guide future modifications needed for this group.

There was no statistically significant difference in the age and gender distribution of the two groups. The waiting time for the CMDC group (median of 89.5 days, IQR=52.8) was significantly shorter than the SOPC group (mean 425 days, s.d. $=220)(P<0.00001)$. All CMDC psychogeriatric patients were referred for psychological intervention. The clinical psychologist appointment was available within 1 month after referral. Only about half of the patients attended the psychological intervention arranged for them. For the SOPC group, 8 patients $(26.7 \%)$ from the SOPC group were referred for psychological intervention with a waiting time of at least 9 months. Two (25\%) refused the intervention. About 60\% of patients in both groups received treatment by general practitioners or private psychiatrists before being referred to us. At 6 months, 28 patients (93\%) remained in CMDC, and 23 patients $(76.7 \%)$ remained in SOPC.
CMDC significantly reduced waiting times for a medical consultation and psychological intervention for elderly patients with CMDs by $80 \%$. The waiting time of psychogeriatric SOPC was also shortened to about 50 weeks after the new programme ran for 10 months. CMDs are common in the elderly population and they are suitable candidates for the clinic. Acceptance towards psychological intervention is only modest for this group of patients as the CMDC programme was not tailor-made for elderly patients. If the clinic is to be extended to psychogeriatric patients, the content of the groups will need to be more age-specific.

Primary care settings present important opportunities for the detection and management of depression in older adults. ${ }^{2}$ Programmes in which primary care providers and mental health specialists collaborate effectively could improve patient outcomes for those with CMDs. ${ }^{3}$ It is worthwhile involving primary care doctors in the new programme by providing care after patients are stepped down from CMDC.

Psychological intervention by nurses helps to reduce the frequency of medical consultations. Doctors' time can thus be spent on less stable patients. Nurse-led clinics were also found to present an opportunity by freeing up specialists to see more complex patients. ${ }^{4}$

Acceptance of psychological intervention may be related to the age group of patients; this information would be useful for guiding future services. Comparison will be performed again when the CMDC group has completed the 1-year programme. A larger number of patients are expected to be discharged from the CMDC in comparison to SOPD after 1 year. The number of doctor consultations will be compared. We hope that by studying the characteristics of patients who can be discharged successfully after completion of the programme, we can identify suitable patients to be referred to it.

Mimi Mei Cheung Wong, Psychiatrist, Pui-fai Pang, Psychiatrist and Michael Gar Chung Yiu, Psychiatrist, United Christian Hospital; Hong Kong email:wmc009@ha.org.hk

1 Common Mental Disorder Clinic, Kowloon West Cluster. Guideline for Common Mental Disorder Clinic Cross-cluster Referral. HAHO-COCGL-Psy-010-V1.

2 Park M, Unützer J. Geriatric depression in primary care. Psychiatr Clin North Am 2011; 34: 469-87.

3 Unützer J, Park M. Strategies to improve the management of depression in primary care. Prim Care 2012; 39: 415-31.

4 Uppal S, Jose J, Banks P, Mackay E. Cost-effective analysis of conventional and nurse-led clinics for common otological procedures. J Laryngol Otol 2004; 118: 189-92.

doi: $10.1192 / p b .41 .5 .298$

\section{Author's reply - the cognitive therapy of depression}

Although Dr Moorey and I both deprecate theoretical whimsy and while there is much that we can agree on concerning cognitive-behavioural therapy (CBT) and psychoanalysis, we profoundly disagree on several other matters including, especially, the content and remit of that paper of mine on which he commented. ${ }^{1,2}$

Regarding CBT, we both agree that it has the most comprehensive evidence base of all the psychological therapies 